\title{
Unexpected donor pulmonary embolism affects early outcomes after lung transplantation: A major mechanism of primary graft failure?
}

Takahiro Oto, MD, Marc Rabinov, FRACS, Anne P. Griffiths, FRCNA, Helen Whitford, FRACP, Bronwyn J. Levvey, RN, Donald S. Esmore, FRACS, Trevor J. Williams, FRACP, and Gregory I. Snell, FRACP

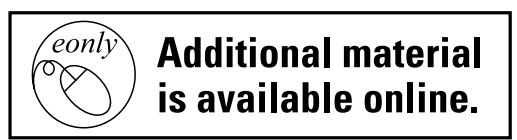

Objective: Primary graft failure remains a significant cause of morbidity and mortality after lung transplantation, and its mechanism is not understood. Previously 2 case reports described fatal primary graft failure due to donor-related unexpected pulmonary embolism. This study investigated the incidence, early outcome, and risk factors of unexpected pulmonary embolism in lung transplantation.

Methods: An exploratory retrograde donor lung flush before implantation to diagnose pulmonary embolism (emboli group) or no pulmonary embolism (no-emboli group) was performed in 74 of 122 consecutive lung transplantations.

Results: The incidence of macroscopic unexpected pulmonary embolism was $38 \%$ ( $28 \%$ clot and $9 \%$ fat). In the emboli group, significantly decreased oxygenation $(P<.05)$, increased pulmonary vascular resistance $(P<.001)$, an increased proportion of opacity on chest radiograph $(P=.03)$, prolonged intubation $(P<$ $.001)$ and intensive care unit stay $(P<.01)$, and decreased 1-year survival $(P=.03)$ were seen after transplantation. In multivariate analysis, pulmonary embolism was an independent risk factor for prolonged intubation (hazard ratio, $2.42 ; P<.01$ ). In logistic regression, death due to trauma with fracture and a smoking history of more than 20 pack-years were significant donor risk factors for pulmonary embolism (adjusted odds ratio, 8.77 and 5.64; $P=.02$ and .04 , respectively). No deleterious effects of the exploratory flush were seen.

Conclusions: Unexpected pulmonary embolism is relatively common, is potentially predicted by donor history (but not by arterial blood gas analysis or chest radiograph), and is associated with primary graft failure. Donor lungs with risk factors of pulmonary embolism should undergo an exploratory flush. When pulmonary embolism is diagnosed, further therapeutic strategies must be considered.

From the Heart and Lung Transplant Unit, The Alfred Hospital, Monash University, Melbourne, Australia.

Received for publication May 26, 2005; revisions received June 30, 2005; accepted for publication July 11, 2005.

Address for reprints: Gregory I. Snell, FRACP, The Alfred Hospital, Heart \& Lung Transplant Unit, Commercial Rd, Melbourne, Victoria 3004, Australia (E-mail: g.snell@ alfred.org.au).

J Thorac Cardiovasc Surg 2005;130:1446-52 $0022-5223 / \$ 30.00$

Copyright $\odot 2005$ by The American Association for Thoracic Surgery

doi:10.1016/j.jtcvs.2005.07.025
$\mathrm{S}$ tandardized donor lung selection criteria have been widely used to ensure quality donor organs for lung transplantation. Recently, extended donor selection criteria have also been used, with acceptable outcomes. ${ }^{1-4}$ However, primary graft failure (PGF) — characterized by pulmonary infiltrates, impaired oxygenation, diminished lung compliance, and diffuse alveolar damage on histologic analysis-remains a significant cause of morbidity and mortality after lung transplantation. The mechanism of PGF has not been elucidated. ${ }^{5-7}$

Previously 2 case reports described fatal PGF that was caused by donor-related pulmonary emboli diagnosed by lung biopsy after transplantation. ${ }^{8,9}$ These case reports suggested that, occasionally, donor selection criteria, which are traditionally based on age, chest radiograph and bronchoscopy findings, respiratory and smoking history, quality and volume of sputum, and blood gas results, might fail to identify donor lungs with a major pathologic process, such as donor-related unexpected pulmonary embolism (PE). Furthermore, the incidence of unexpected PE in donor 

Abbreviations and Acronyms
$\mathrm{CPB}=$ cardiopulmonary bypass
$\mathrm{FIO}_{2}=$ inspired oxygen fraction
ICU $=$ intensive care unit
$\mathrm{PE}=$ pulmonary embolism
$\mathrm{PGF}=$ primary graft failure
$\mathrm{PVR}=$ pulmonary vascular resistance

lungs and the outcome of lungs with PE after lung transplantation remain unknown because of the difficulty of detecting PE, especially before transplantation.

To further explore the relationship of unexpected PE with PGF, we used a simple exploratory method of a brief retrograde donor lung flush before implantation. The aims of this study were to describe the incidence of unexpected $\mathrm{PE}$ in donor lungs that were accepted for transplantation, to investigate the early outcomes after lung transplantation, and to investigate the risk factors for unexpected PE.

\section{Materials and Methods}

From April 2002 to April 2005, a total of 122 consecutive lung transplantations, including 86 bilateral single-lung and 36 singlelung transplantations, were performed at the Alfred Hospital, and all of these were included in this study.

\section{Donor and Recipient Assessment and Matching}

Although lung donor selection criteria are based on standard criteria, extended donors are commonly used at our institution. ${ }^{2,6,10,11}$ Preoperative immunologic evaluation was routinely performed. The presence of preformed antibodies to human leukocyte antigen was screened with a panel-reactive antibody assay, and all transplant recipients were found to have values less than $10 \%$. A prospective donor-recipient T-cell and B-cell cross-match was performed in all cases.

\section{Lung Procurement}

Lung procurement and preservation basically followed standard procedures. ${ }^{12-14}$ This included an intravenous infusion of prostacyclin (Flolan) at 40 to $80 \mathrm{ng} \cdot \mathrm{kg}^{-1} \cdot \mathrm{min}^{-1}$ for approximately 10 minutes before crossclamping, followed by a single antegrade flush with cold modified Euro-Collins solution $(60 \mathrm{~mL} / \mathrm{kg})$. After September 2004, Perfadex (Vitrolife, Göteborg, Sweden) replaced Euro-Collins solution at our institution.

\section{Exploration of Unexpected PE}

We used a simple exploratory method to detect unexpected PE in donor lungs. ${ }^{15}$ Before implantation, a brief retrograde flush was performed via pulmonary venous cuff on a back table. Approximately 50 to $70 \mathrm{~mL}$ of cold Ringer solution was gently flushed by a selectively cannulated ball syringe for each pulmonary venous segmental branch. Details of macroscopic PEs flushed out of the pulmonary arteries were recorded. When any PE was detected, a brief flush was repeated until no further PE was flushed out. An exploratory flush became routine practice in our institution in
February 2004. Before then, exploratory flushing was performed when indicated by the appearance of possible small pulmonary infarcts or contusions in the donor lung, protracted donor inpatient stay, the surgeon's preference, and donors who died of, especially, multiple traumas.

\section{Transplantation Procedure}

Cardiopulmonary bypass (CPB) was not routinely performed in our institution except for pulmonary hypertension. CPB was considered in cases of intolerance of single-lung ventilation due to hemodynamic instability. ${ }^{12}$ Before completion of implantation, retrograde reperfusion and deairing was performed via an untied pulmonary arterial anastomotic suture line. A specific pressureand flow-controlled technique was not used. This was then followed by antegrade reperfusion.

\section{Postoperative Management}

Postoperative management in the intensive care unit (ICU) was performed to ensure satisfactory end-organ perfusion while a relatively low filling pressure was maintained (cardiac index $>2.4$, pulmonary capillary wedge pressure $<10 \mathrm{~mm} \mathrm{Hg}$, and central venous pressure $<7 \mathrm{~mm} \mathrm{Hg}$ ). Patients with PGF received a standardized evaluation and therapy with increasing complexity depending on the degree of ventilatory and hemodynamic compromise. $^{6,16}$

\section{Immunosuppression and Surveillance of Transbronchial Lung Biopsy}

Immunosuppression was based on triple therapy with cyclosporine (trough levels of 300-450 $\mu \mathrm{g} / \mathrm{L}$ ), azathioprine (1.5-2.0 mg . $\left.\mathrm{kg}^{-1} \cdot \mathrm{d}^{-1}\right)$, and prednisolone $\left(0.15 \mathrm{mg} \cdot \mathrm{kg}^{-1} \cdot \mathrm{d}^{-1}\right)$. Prophylaxis for Pneumocystis carinii and cytomegalovirus infection was achieved with low-dose oral trimethoprim-sulfamethoxazole and intravenous and oral ganciclovir, respectively. Surveillance bronchoscopy and transbronchial lung biopsy were performed at $0.5,1,2,3,6,9,12,18$, and 24 months and yearly thereafter. Acute rejection was defined and treated according to standard criteria. ${ }^{3,16,17}$

\section{Data Collection}

Data regarding PE were recorded prospectively; however, other data were retrieved retrospectively from the transplant database and from a review of ICU and donor records. Arterial blood gas analysis data and pulmonary vascular resistance (PVR) data were collected at $0,6,12,18$, and 24 hours after admission to the ICU. The donor $\mathrm{PaO}_{2}$ was the last arterial blood gas result on an inspired oxygen fraction $\left(\mathrm{FIO}_{2}\right)$ of 1.0 and a positive end-expiratory pressure of $5 \mathrm{~cm} \mathrm{H}_{2} \mathrm{O}$ recorded at the time of donor referral to the transplantation center. The graft ischemic time of a bilateral singlelung transplantation was defined as the ischemic time for the second transplanted lung. The degree of opacification on the chest radiograph at the time of donor referral and 24 hours after transplantation was assessed and scored by 2 blinded reviewers as follows: clear, no infiltrate; mild, mild interstitial infiltrate in less than 1 lobe; and moderate/severe, moderate to severe interstitial infiltrate in more than 1 lobe or extensive alveolar infiltrate. 

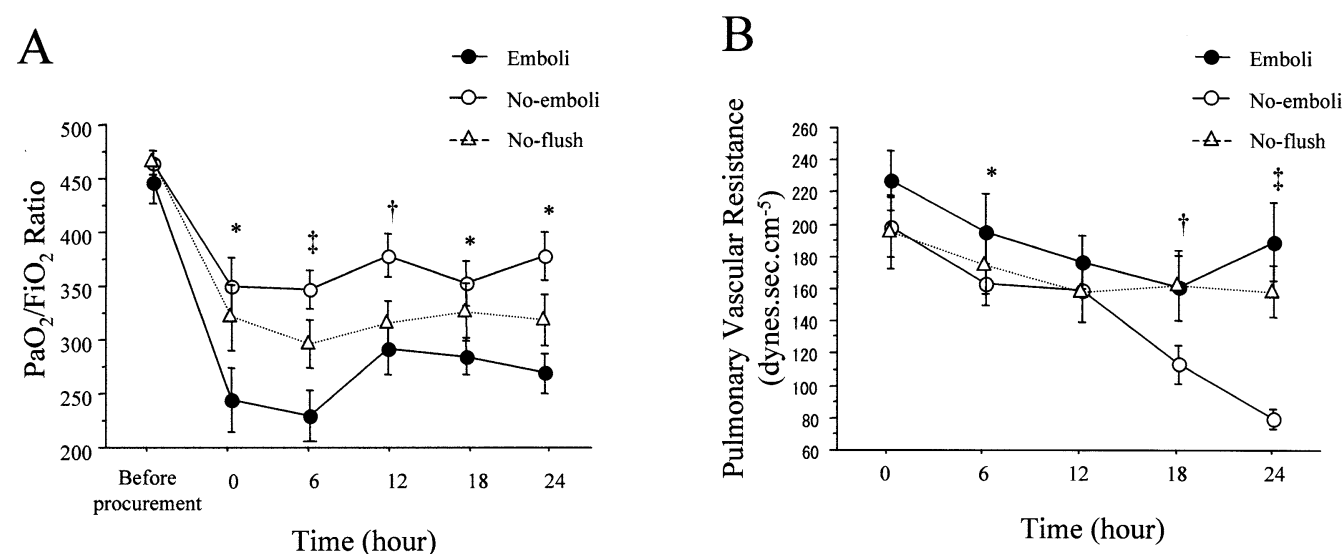

Figure 1. A, Arterial oxygen tension/inspired oxygen consumption ratio $\left(\mathrm{PaO}_{2} / \mathrm{FiO}_{2}\right.$ ratio) before and after transplantation. B, Pulmonary vascular resistance in the first 24 hours after transplantation. Comparison was made between the emboli and no-emboli groups. ${ }^{*} P<.05 ;+P<.01 ; ¥ P<.001$.

\section{Assessment of Outcome}

The $\mathrm{PaO}_{2} / \mathrm{FIO}_{2}$ ratio, $\mathrm{PVR}$, chest radiograph, duration of intubation, length of ICU stay, time free from acute rejection, lung function 3 months after transplantation, and 1-year survival were used as outcome indicators.

\section{Study Groups}

First, we divided the recipients who received lungs with an exploratory flush (exploratory flush group) and recipients who received lungs without an exploratory flush (no-flush group). Second, to investigate the incidence and effect of PE, we divided the exploratory flush group into the following 2 groups according to the result of the exploratory flush: recipients who received lungs with PE that included clot and fat (emboli group) and recipients who received lungs without any PE (no-emboli group). Third, the emboli group was subgrouped by the material of the PE.

\section{Statistical Analysis}

Analysis was performed with the StatView 5.0 software package (SAS Institute, Inc, Cary, NC). Continuous data were initially assessed for normality and expressed as mean $\pm \mathrm{SE}$; categorical data were expressed as count and proportions. Comparison between groups was performed with the $\chi^{2}$ test for categorical variables, with the Student $t$ test for parametric continuous variables, and with the Mann-Whitney $U$ test for nonparametric continuous variables, if appropriate. Repeated-measures variables, including the $\mathrm{PaO}_{2} / \mathrm{FIO}_{2}$ ratio and PVR, were analyzed with 2-way repeated-measures analysis of variance. Duration of intubation, length of ICU stay, time to first acute rejection, and 1-year survival were estimated by the Kaplan-Meier method, and the curves were analyzed by using the log-rank test. Univariate and multivariate analysis for duration of intubation, length of ICU stay, and 1-year survival were performed by using Cox proportional hazards regression, and risk factors for PE were analyzed with logistic regression. All possible prediction variables were put into the univariate analysis, including donor age, sex, cause of death, history of smoking, oral contraceptive/hormone-replacement therapy use, preprocurement intubation time, prophylactic anticoagulant therapy, last $\mathrm{PaO}_{2}$, and $\mathrm{PE}$ in donor lungs, as well as recipient age, sex, underlying disease, type and year of transplantation, graft ischemic time, and use of CPB. Risk factors with a level of significance defined as $P<.20$ in univariate analysis were adjusted in the multivariate analysis.

\section{Results}

Incidence and Character of Unexpected PE

An exploratory flush was performed for 74 of 122 lung transplants (Table E1; see online-only materials). PE was detected in 28 of 74 donors, and the incidence of PE was $38 \%$. Twenty-one of 28 PEs were clot emboli, and 7 were fat emboli. The demographics of donors and recipients were similar between the emboli and no-emboli groups except for CPB use $(P<.05)$. In the emboli group, 7 patients required CPB because of preexisting pulmonary hypertension, and 2 required it because of intolerance of single-lung ventilation during the second lung implantation.

\section{Oxygenation Before and After Transplantation}

The $\mathrm{PaO}_{2} / \mathrm{FIO}_{2}$ ratio before procurement and after transplantation is depicted in Figure 1, A. Although the $\mathrm{PaO}_{2} / \mathrm{FIO}_{2}$ ratio before procurement was similar between groups, the $\mathrm{PaO}_{2} / \mathrm{FIO}_{2}$ ratio in the emboli group was significantly worse than that in the no-emboli group throughout the first 24 hours after admission to the ICU.

\section{Pulmonary Vascular Resistance}

PVR in the no-emboli group decreased constantly in the first 24 hours after admission to the ICU; however, the PVR in the emboli group remained significantly higher than that in the no-emboli group (Figure 1,B). 

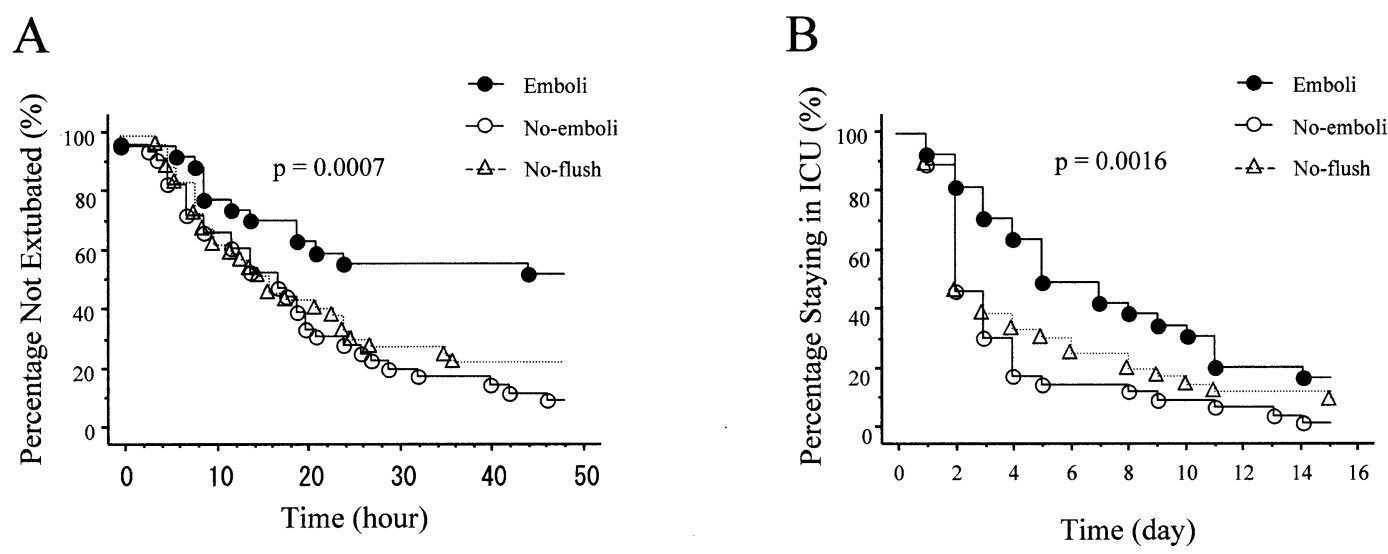

Figure 2. A, Percentage of patients not extubated versus time after transplantation. B, Percentage of patients staying in the intensive care unit versus time after transplantation. Comparison was made between the emboli and no-emboli groups.

\section{Chest Radiograph}

The relative proportion of opacity scores of donor chest radiographs was similar between groups $(P=.52)$; approximately $60 \%$ of donors had a clear chest radiograph (Figure $\mathrm{E} 1, A)$. After transplantation, an increased proportion in mild to severe opacity on chest radiograph categories was seen in the emboli group, but not in the no-emboli group $(P=.03)$. The percentage of clear chest radiographs seen in the emboli group was only $7 \%$, in contrast to $43 \%$ in the no-emboli group (Figure 1, $B$ ).

\section{Duration of Intubation}

The percentage of patients not extubated versus time after lung transplantation was estimated by the Kaplan-Meier method (Figure 2, A). The patients in the emboli group remained intubated significantly longer than those in the no-emboli group $(P=.0007)$ : the percentage of patients who remained intubated in the emboli group was 54\%, compared with $9 \%$ in the no-emboli group, even 48 hours after admission to the ICU.

\section{Length of ICU Stay}

The length of ICU stay in the emboli group was significantly longer than that in the no-emboli group (Figure 2, B; $P=.0016)$. The percentage of patients who stayed in the ICU longer than 7 days was $42 \%$ in the emboli group and $15 \%$ in the no-emboli group.

\section{Acute Rejection}

The percentage of patients with International Society for Heart and Lung Transplantation grade A0 diagnosed by the first transbronchial lung biopsy performed 2 weeks after transplantation was 50\% in the emboli group and 39\% in the no-emboli group $(P=.11)$. There was no significant dif- ference in time to first acute rejection (International Society for Heart and Lung Transplantation grade A2) between groups $(P=.34)$.

\section{Lung Function 3 Months After Transplantation}

The predicted forced expiratory volume in 1 second 3 months after transplantation was similar between the emboli and no-emboli groups $(74 \% \pm 4 \%$ and $78 \% \pm 5 \%$, respectively; $P=.62$ ).

\section{One-Year Survival}

Thirty-day, 90-day, and 1-year survival in the emboli and no-emboli groups were $93 \%$ and $100 \%, 89 \%$ and $100 \%$, and $85 \%$ and $97 \%$, respectively (Figure E2). An early drop of the survival curve in emboli group was seen $(P=.03)$.

\section{Univariate and Multivariate Analysis}

Cox proportional hazard regression was constructed for the duration of intubation, length of ICU stay, and 1-year survival. In the multivariate analysis, PE was an independent and significant risk factor for prolonged intubation after transplantation (hazard ratio, 2.42; 95\% confidence interval, 1.25-4.70; $P<.01$; Table 1); however, no factors reached statistical significance for length of ICU stay or 1-year survival.

\section{Subgroup Analysis of PE}

In univariate analysis, both clot and fat embolism were significant risk factors for prolonged intubation (hazard ratio, 0.44 and 0.16 ; $95 \%$ confidence interval, $0.22-0.80$ and $0.04-0.67 ; P=.02$ and .01 , respectively) and ICU stay (hazard ratio, 0.52 and $0.37 ; 95 \%$ confidence interval, 0.29 0.93 and $0.15-0.89 ; P=.03$ and .03 , respectively). There- 
TABLE 1. Cox proportional hazard regression for duration of intubation

\begin{tabular}{lccr}
\hline Variable & $\begin{array}{c}\text { Hazard } \\
\text { ratio }\end{array}$ & $\mathbf{9 5 \%} \mathbf{~ I ~}$ & $\boldsymbol{P}$ value \\
\hline Univariate analysis & & & \\
$\quad$ Pulmonary embolism & 2.89 & $1.51-5.56$ & $<.01$ \\
Smoking $>$ 20 pack-y & 2.22 & $1.02-4.84$ & .04 \\
CPB use & 5.84 & $2.34-14.55$ & $<.01$ \\
Underlying disease & & & \\
$\quad$ COPD & 1.00 & Reference & Reference \\
$\quad$ CF & 1.02 & $0.63-1.66$ & .94 \\
$\quad$ ILD & 0.46 & $0.21-0.99$ & .04 \\
$\quad$ PH & 0.14 & $0.03-0.57$ & $<.01$ \\
Bilateral single & & & \\
$\quad$ transplantation & 0.54 & $0.32-0.90$ & .02 \\
Multivariate analysis & & & \\
$\quad \begin{array}{l}\text { Pulmonary embolism } \\
\text { CPB use }\end{array}$ & 2.42 & $1.25-4.70$ & $<.01$ \\
& 5.98 & $1.47-24.40$ & .02 \\
\hline
\end{tabular}

$\mathrm{Cl}$, Confidence interval; $C O P D$, chronic obstructive pulmonary disease; $C F$, cystic fibrosis; ILD, interstitial lung disease; $P H$, pulmonary hypertension; $C P B$, cardiopulmonary bypass.

fore, on the basis of this hazard ratio, fat embolism seemed to represent a higher risk factor than did clot embolism.

\section{Risk Factors for PE}

Logistic regression analysis of risk factors for PE is shown in Table 2. Donor death due to trauma with fracture and a smoking history of more than 20 pack-years were significant risk factors for PE (adjusted odds ratio, 8.77 and 5.64; 95\% confidence interval, 1.37-51.21 and 1.06-30.00; $P=$ .02 and .04 , respectively).

\section{Effect of Exploratory Flush}

There was no significant difference between the exploratory flush and no-flush groups in donor and recipient demographics except for the type of transplantation (Table E1; $P<.01)$. In every posttransplantation outcome variable- including oxygenation, PVR, chest radiograph appearance, duration of intubation, length of ICU stay, and 1-year survival-the mean value in the no-flush group tended to be between that in the emboli and no-emboli groups.

\section{Discussion}

We have described a detailed assessment of unexpected donor PE in lung transplantation. A deleterious effect from unexpected PE was shown in early posttransplantation oxygenation, PVR, chest radiograph, duration of intubation, length of ICU stay, and 1-year survival. In multivariate analysis, unexpected PE was independently and strongly associated with prolonged intubation after transplantation.

PGF is clinically recognized as impaired gas exchange, opacification of the chest radiograph, and increased PVR within 24 hours of transplantation and results in the requirement for prolonged mechanical ventilatory support with high levels of inspired oxygen and a prolonged stay in the ICU.

Many factors, including donor, recipient, and operative factors, are reported as associated with PGF. ${ }^{5-7,18}$ However, no previous prospective study has shown a statistical association of unexpected PE with PGF. It is important to note that the findings of this study might explain a significant proportion of previously unexplained PGF in lung transplantation.

The deleterious effect of unexpected PE on lung performance is, in part, due to mechanical obstruction. Pulmonary arterial obstruction augments alveolar dead space and ventilation/perfusion mismatch, thus resulting in impaired gas exchange. ${ }^{19}$ At the time of procurement, ventilation/perfusion mismatch might be autogenously corrected by reducing alveolar dead space with reflex bronchoconstriction and vasoconstriction and maintaining oxygenation and, thus, the $\mathrm{PaO}_{2} / \mathrm{FIO}_{2}$ ratio. However, after transplantation, denervated lungs might not be able to correct a ventilation/perfusion mismatch; this would result in impaired oxygenation in the emboli group (Figure 1,A). Additionally, the deleterious effect of PE on the lungs might be due to its inflammatory

TABLE 2. Logistic regression analysis of risk factors for pulmonary embolism

\begin{tabular}{|c|c|c|c|}
\hline Variable & Unadjusted odds ratio $(95 \% \mathrm{CI})$ & Adjusted odds ratio $(95 \% \mathrm{Cl})$ & $P$ value \\
\hline Donor cause of death & & & \\
\hline $\begin{array}{l}\text { Trauma } \\
\text { With fracture }\end{array}$ & $8.25(1.45-86.83)$ & $8.77(1.37-51.21)$ & .02 \\
\hline Without fracture & $1.53(0.29-7.94)$ & $2.26(0.39-13.11)$ & .36 \\
\hline CVA (hemorrhagic) & $1.96(0.42-9.11)$ & $1.31(0.23-7.49)$ & .74 \\
\hline CVA (thrombotic) & $1.22(0.09-16.59)$ & $0.44(0.02-11.39)$ & .62 \\
\hline Anoxia & 1.00 & 1.00 & Reference \\
\hline Others & $7.33(0.48-111.11)$ & $6.44(0.34-120.90)$ & .21 \\
\hline Smoking $>20$ pack-y & $3.50(0.92-13.30)$ & $5.64(1.06-30.00)$ & .04 \\
\hline Oral contraceptives/HRT & $4.78(0.86-26.58)$ & $9.08(0.80-103.54)$ & .08 \\
\hline
\end{tabular}

$\mathrm{Cl}$, Confidence interval; CVA, cerebrovascular accident; HRT, hormone-replacement therapy. 
effect on the endothelium of the pulmonary artery. PE might activate local inflammatory mediators: this would exaggerate ischemia/reperfusion injury and increase endothelial permeability. In turn, this would lead to increased interstitial infiltration, with resultant opacity on chest radiograph (seen in $40 \%$ of patients before and in $93 \%$ after transplantation in the emboli group; Figure E1), and also to impaired oxygenation and increased PVR (Figure 1). This inflammatory response seems to be a nonalloimmune response, because no effect was found in this study on acute rejection in the first lung biopsy, time to acute rejection, and lung function 3 months after transplantation. Further study is appropriate as local endothelial dysfunction, exaggerated by unexpected PE, may be important in the pathogenesis of PGF.

It is important to consider whether the presence of $\mathrm{PE}$ in the donor, with its related poor early outcome, can be predicted prospectively. In this study, donor death due to trauma with fracture and a donor smoking history of more than 20 pack-years were substantial risk factors for unexpected PE (Table 2). Fracture is a common risk factor for fat embolism, and heavy smoking, trauma, drug use, cancer, chemotherapy, oral contraceptives, and hormonereplacement therapy are well-recognized risk factors for deep venous thrombosis and PE. ${ }^{19,20}$ In this study, which primarily consisted of trauma and hemorrhagic cerebral vascular accident donors, very few received anticoagulant therapy because of concerns of further hemorrhage. Consequently, compared with other potential organ donors, trauma patients with fractures have a greater risk of thromboemboli and fat emboli.

The $\mathrm{PaO}_{2} / \mathrm{FIO}_{2}$ ratio before procurement in this study was similar between the emboli and no-emboli groups. Previous literature regarding suspected $\mathrm{PE}$ in the nontransplantation population suggested that arterial blood gas analysis was not specific and did not rule out the diagnosis of PE; also, approximately $20 \%$ of patients with angiographically proven PE had a normal alveolar/arterial oxygen gradient. ${ }^{21}$ Furthermore, previous transplantation literature also indicates that even organ donors who died as a result of acute $\mathrm{PE}$ could have an excellent $\mathrm{PaO}_{2} / \mathrm{FIO}_{2}$ ratio at the time of procurement. ${ }^{22}$ Arterial blood gas analysis was not predictive for unexpected PE in donor lungs.

In this study, the chest radiograph before procurement seemed unhelpful in discriminating donors with PE from other potential donors. Previous articles regarding imaging studies for PE in the nontransplantation population suggested that chest radiographs could not be used to diagnose or exclude PE. ${ }^{19}$

Regarding abnormal macroscopic lung findings at the time of procurement, Smith and colleagues ${ }^{23}$ reported a maldistribution of preservation solution on lungs with PE during flushing of the pulmonary arteries. In contrast, Nguyen and colleagues ${ }^{24}$ reported that there was no evi- dence of maldistribution of the preservation solution in a donor with PE. Unfortunately, these case reports included only 1 donor in each case. A maldistribution of preservation solution throughout the lungs is not rare regardless of the presence of PE and might be associated with other circumstances, including poorly ventilated areas, lung contusions, or local vasoconstriction. ${ }^{25}$ The authors of these 2 case reports also described successful thromboembolectomy for unexpected $\mathrm{PE}$ at the time of donor lung preparation before implantation. However, in this study, unexpected PE could be detected only by direct vision through the pulmonary arterial cuff in 2 of 28 donors in the emboli group; otherwise, PE was isolated to the subsegmental pulmonary arteries or more distal branches. Isolated PE of the subsegmental pulmonary arteries is not unusual and occurred in $30 \%$ of patients with $\mathrm{PE}$ in a previous nontransplantation pulmonary angiographic study. ${ }^{26} \mathrm{We}$ believe that an exploratory flush may be the only diagnostic method of detecting unexpected PE isolated in subsegmental pulmonary arteries.

In comparison between the exploratory flush and noflush groups, no direct effect of the exploratory flush itself on lungs was seen. In other words, the exploratory flush seemed not to either improve or exacerbate lung function. Therefore, the exploratory flush seems to function as a diagnostic method and not as a treatment. Further therapeutic strategies might be warranted for patients in emboli group.

To remove the residual emboli, a retrograde full-dose flush with mechanical ventilation might be beneficial. ${ }^{14,15,27}$ Venuta and associates ${ }^{15}$ observed blood, clot, and fat in the retrograde perfusate in all 7 patients described in the literature of their clinical experience of preimplantation retrograde preservation flush $(30-40 \mathrm{~mL} / \mathrm{kg})$. de Perrot and Keshavjee $^{14}$ mentioned the potential of retrograde preservation flush to remove residual blood, clot, and PE. The endothelium of the pulmonary artery with PE already damaged at the time of procurement is aggravated by maldistribution of preservation solution, and this has implications for ischemia/reperfusion injury. Retrograde flush at the time of procurement might have a more protective effect on damaged endothelium (by uniform distribution of flush solution) than that at the time of implantation. However, the routine application of a large-volume retrograde flush might not be necessary, because patients without PE in this study had satisfactory outcomes without a large-volume retrograde flush.

Previously, 2 case reports described successful use of lungs from fatal acute PE donors treated by thrombolysis several days before organ procurement. ${ }^{22,28}$ Dissolving and removing PEs is effective; however, careful consideration is necessary when thrombolysis is applied to lungs with unexpected PE because of the risk of postoperative bleeding.

Other therapeutic strategies to prevent ischemia/reperfusion injury, such as using complement inhibitor ${ }^{29}$ and antagonist of platelet-activating factor, ${ }^{30}$ have been investi- 
gated by clinical trials and are reported to be useful. An exploratory flush could distinguish specific donor lungs at high risk of early graft dysfunction that would require further therapeutic approaches. This may contribute to better patient outcomes with cost-effectiveness.

A possible limitation to this study should be considered. Data regarding the result of exploratory flush were collected prospectively; however, this study had prospective and retrospective components, and, therefore, there might be a potential bias in selecting patients into the flush or no-flush groups. The incidence of PE between February 2004 and April 2005, when exploratory flush was routinely performed, was 38\% (18/47) — equal to the overall incidence of PE $(38 \%$; 28/74). Although the incidence of PE in the no-flush group was unknown, in every posttransplantation outcome variable the mean value in the no-flush group tended to be between that in the emboli and no-emboli groups. This result supports the notion that there was likely to be a similar distribution of patients with emboli between the flush and no-flush groups.

In conclusion, this is the first study that has described a detailed analysis of donor-related unexpected PE in lung transplantation. The incidence of unexpected PE is high. Unexpected PE is potentially predicted by a donor history of trauma with fracture and smoking more than 20 packyears-not by donor arterial blood gas analysis or chest radiograph. Unexpected PE is associated with PGF. Therefore, donors with risk factors for unexpected PE should be considered as marginal donors for lung transplantation. All the lung grafts of donors with risk factors of unexpected PE should undergo an exploratory flush. When PE is diagnosed, further therapeutic strategies must be considered.

\section{References}

1. Orens JB, Boehler A, de Perrot M, Estenne M, Glanville AR, Keshavjee $\mathrm{S}$, et al. A review of lung transplant donor acceptability criteria. J Heart Lung Transplant. 2003;22:1183-200.

2. Gabbay E, Williams TJ, Griffiths AP, Macfarlane LM, Kotsimbos TC, Esmore DS, et al. Maximizing the utilization of donor organs offered for lung transplantation. Am J Respir Crit Care Med. 1999;160:265-71.

3. Trulock EP, Edwards LB, Taylor DO, Boucek MM, Keck BM, Hertz MI. The registry of the International Society for Heart and Lung Transplantation: twenty-first official adult lung and heart-lung transplant report-2003. J Heart Lung Transplant. 2004;23:804-15.

4. de Perrot M, Snell GI, Babcock WD, Meyers BF, Patterson GA, Hodges TN, et al. Strategies to optimize the use of currently available lung donors. J Heart Lung Transplant. 2004;23:1127-34.

5. Christie JD, Kotloff RM, Pochettino A, Arcasoy SM, Rosengard BR, Landis JR, et al. Clinical risk factors for primary graft failure following lung transplantation. Chest. 2003;124:1232-41.

6. Oto T, Levvey B, Pilcher DV, Bailey MJ, Snell GI. Evaluation of the oxygenation ratio in the definition of early graft dysfunction post lung transplantation. J Thorac Cardiovasc Surg. 2005;130:180-6.

7. Thabut G, Vinatier I, Stern JB, Lesèche G, Loirat P, Fournier M, et al. Primary graft failure following lung transplantation: predictive factors of mortality. Chest. 2002;121:1876-82.

8. Waller DA, Bennett MK, Corris PA, Dark JH. Donor-acquired fat embolism causing primary organ failure after lung transplantation. Ann Thorac Surg. 1995;59:1565-6.
9. Rosendale BE, Keenan RJ, Duncan SR, Hardesty RL, Armitage JA, Griffith BP, et al. Donor cerebral emboli as a cause of acute graft dysfunction in lung transplantation. J Heart Lung Transplant. 1992; 11:72-6.

10. Oto T, Griffiths A, Levvey B, Whitford H, Kotsimbos T, Rabinov M, et al. Donor history of asthma is not a contraindication to lung transplantation: 12-year single center experience. J Heart Lung Transplant. 2004;23:309-16.

11. Oto T, Griffiths AP, Levvey B, Pilcher DV, Whitford H, Kotsimbos TC, et al. A donor history of smoking affects early but not late outcome from lung transplantation. Transplantation. 2004;78:599-606.

12. Oto T, Rabinov M, Rosenfeldt F, Esmore DS. Extended pericardiotomy avoids cardiopulmonary bypass during bilateral sequential lung transplantation. J Thorac Cardiovasc Surg. 2005;129:466-7.

13. Oto T, Rosenfeldt F, Rowland M, Pick A, Rabinov M, Preovolos A, et al. Extracorporeal membrane oxygenation after lung transplantation: evolving technique improves outcomes. Ann Thorac Surg. 2004;78: 1230-5.

14. de Perrot, Keshavjee S. Lung preservation. Semin Thorac Cardiovasc Surg. 2005;16:300-8.

15. Venuta F, Rendina EA, Bufi M, Della Rocca G, De Giacomo T, Gabriella Costa M, et al. Preimplantation retrograde pneumoplegia in clinical lung transplantation. J Thorac Cardiovasc Surg. 1999;118: 107-14.

16. Trulock EP. Management of lung transplant rejection. Chest. 1993; 103:1566-76.

17. Snell GI, Bennetts K, Bartolo J, Levvey B, Griffiths A, Williams T, et al. Body mass index as a predictor of survival in adults with cystic fibrosis referred for lung transplantation. J Heart Lung Transplant. 1998;17:1097-103.

18. Lee KH, Martich GD, Boujoukos AJ, Keenan RJ, Griffith BP. Predicting ICU length of stay following single lung transplantation. Chest. 1996;110:1014-7.

19. Goldhaber SZ. Pulmonary embolism. Lancet. 2004;363:1295-305.

20. Lisse JR, Davis CP, Thurmond-Anderle M. Cocaine abuse and deep venous thrombosis. Ann Intern Med. 1989;110:571-2.

21. Stein PD, Goldhaber SZ, Henry JW, Miller AC. Arterial blood gas analysis in the assessment of suspected acute pulmonary embolism. Chest. 1996;109:78-81.

22. Fischer S, Gohrbandt B, Meyer A, Simon AR, Haverich A, Strúber M. Should lungs from donors with severe acute pulmonary embolism be accepted for transplantation? The Hannover experience. J Thorac Cardiovasc Surg. 2003;126:1641-3.

23. Smith JA, Mohajeri M, Rabinov M, Esmore DS. Maldistribution of pneumoplegia in pulmonary allograft secondary to post-traumatic pulmonary thromboembolism. J Heart Lung Transplant. 1996;15:324-5.

24. Nguyen DQ, Salerno CT, Bolman M III, Park SJ. Pulmonary thromboembolectomy of donor lung prior to lung transplantation. Ann Thorac Surg. 1999;67:1787-9.

25. Baretti R, Bitu-Moreno J, Beyersdorf F, Matheis G, Francischetti I, Kreitmair B. Distribution of lung preservation solution in parenchyma and airways: influence of atelectasis and route of delivery. $J$ Heart Lung Transplant. 1995;14:80-91.

26. Oser RF, Zukerman DA, Gutierrez FR, Brink JA. Anatomic distribution of pulmonary emboli at pulmonary angiography: implications for cross-sectional imaging. Radiology. 1996;199:31-5.

27. Wittwer T, Franke UW, Fehrenbach A, Ochs M, Sandhaus T, Schutte A, et al. Experimental lung transplantation: impact of preservation solution and route of delivery. J Heart Lung Transplant. In press.

28. Frenia D, Nathan SD, Ahmad S, Guerreo M, Distefano D, Massamiano $\mathrm{P}$, et al. Successful lung transplantation from a donor with a saddle pulmonary embolus. J Heart Lung Transplant. In press.

29. Keshavjee S, Davis RD, Zamora MR, de Perrot M, Patterson GA. A randomized, placebo controlled trial of complement inhibition in ischemia-reperfusion injury after lung transplantation in human beings. J Thorac Cardiovasc Surg. 2005;129:423-8.

30. Wittwer T, Grote M, Oppelt P, Franke U, Schaefers HJ, Wahlers T. Impact of PAF antagonist BN 52021 (Ginkolide B) on post-ischemic graft function in clinical lung transplantation. J Heart Lung Transplant. 2001;20:358-63. 
TABLE E1. Demographics of donor and recipient

\begin{tabular}{|c|c|c|c|c|c|}
\hline \multirow[b]{2}{*}{ Variable } & \multicolumn{3}{|c|}{ Exploratory flush $(n=74)$} & \multirow[b]{2}{*}{$\begin{array}{l}\text { No flush } \\
(\mathrm{n}=48)\end{array}$} & \multirow[b]{2}{*}{$P$ value } \\
\hline & $\begin{array}{l}\text { Emboli } \\
(n=28)\end{array}$ & $\begin{array}{l}\text { No emboli } \\
(n=46)\end{array}$ & $P$ value & & \\
\hline \multicolumn{6}{|l|}{ Donor } \\
\hline Age, y (mean $\pm S E)$ & $33 \pm 3$ & $38 \pm 2$ & $>.2$ & $36 \pm 2$ & $>.2$ \\
\hline Sex & & & $>.2$ & & $>.2$ \\
\hline Male & 20 & 31 & & 31 & \\
\hline Female & 8 & 15 & & 17 & \\
\hline Oral contraceptives/HRT & 4 & 2 & $>.2$ & & \\
\hline Smoking $>20$ pack-y & 6 & 4 & $>.2$ & 6 & $>.2$ \\
\hline Cause of death & & & $>.2$ & & $>.2$ \\
\hline \multicolumn{6}{|l|}{ Trauma } \\
\hline With fracture & 9 & 4 & & 8 & \\
\hline Without fracture & 5 & 12 & & 11 & \\
\hline \multicolumn{6}{|l|}{ CVA } \\
\hline Hemorrhagic & 8 & 15 & & 15 & \\
\hline Thrombotic & 1 & 3 & & 5 & \\
\hline Anoxia & 3 & 11 & & 7 & \\
\hline Others & 2 & 1 & & 2 & \\
\hline Anticoagulant therapy & 1 & 5 & $>.2$ & 2 & $>.2$ \\
\hline Preprocurement intubation time, $\mathrm{h}$ (mean $\pm \mathrm{SE}$ ) & $53 \pm 6$ & $61 \pm 9$ & $>.2$ & $61 \pm 5$ & $>.2$ \\
\hline Preprocurement $\mathrm{PaO}_{2} / \mathrm{F}_{10}$ & $455 \pm 21$ & $474 \pm 12$ & $>.2$ & $474 \pm 12$ & $>.2$ \\
\hline \multicolumn{6}{|l|}{ Recipient } \\
\hline Age, y (mean $\pm S E)$ & $45 \pm 3$ & $47 \pm 2$ & $>.2$ & $48 \pm 2$ & $>.2$ \\
\hline Sex & & & $>.2$ & & $>.2$ \\
\hline Male & 17 & 33 & & 30 & \\
\hline Female & 11 & 13 & & 18 & \\
\hline Underlying disease & & & & & $>.2$ \\
\hline COPD & 11 & 21 & & 19 & \\
\hline $\mathrm{CF}$ & 8 & 18 & & 16 & \\
\hline ILD & 4 & 5 & & 11 & \\
\hline $\mathrm{PH}$ & 5 & 2 & & 2 & \\
\hline Type of transplantation & & & $>.2$ & & $<.01$ \\
\hline Bilateral & 24 & 35 & & 27 & \\
\hline Single & 4 & 11 & & 21 & \\
\hline CPB use & 9 & 6 & $<.05$ & 6 & $>.2$ \\
\hline Ischemic time, min (mean $\pm \mathrm{SE}$ ) & $368 \pm 26$ & $370 \pm 20$ & $>.2$ & $379 \pm 19$ & $>.2$ \\
\hline
\end{tabular}

$H R T$, Hormone-replacement therapy; $C V A$, cerebrovascular accident; $P a O_{2} / F I O_{2}$, ratio of arterial oxygen tension and inspired oxygen fraction; $C O P D$, chronic obstructive pulmonary disease; $C F$, cystic fibrosis; $I L D$, interstitial lung disease; $P H$, pulmonary hypertension; $C P B$, cardiopulmonary bypass. 

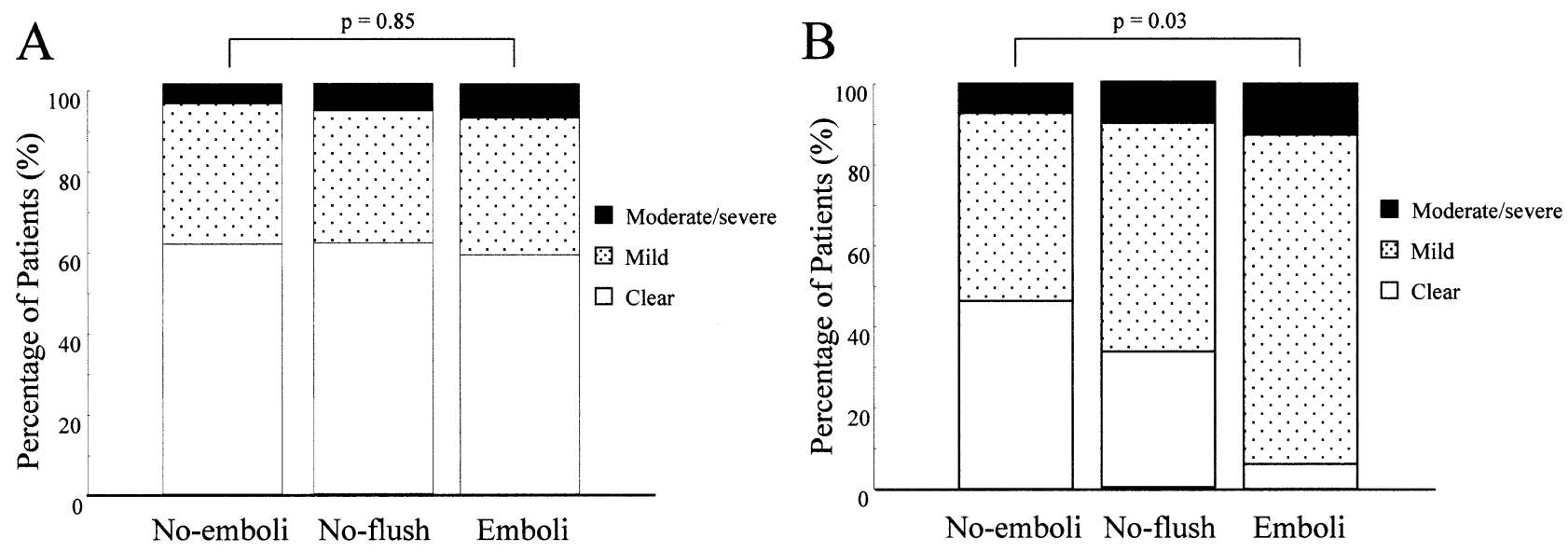

Figure E1. A, Percentage of donor chest radiographs showing infiltrate before procurement. B, Percentage of recipient chest radiographs showing infiltrate 24 hours after transplantation.

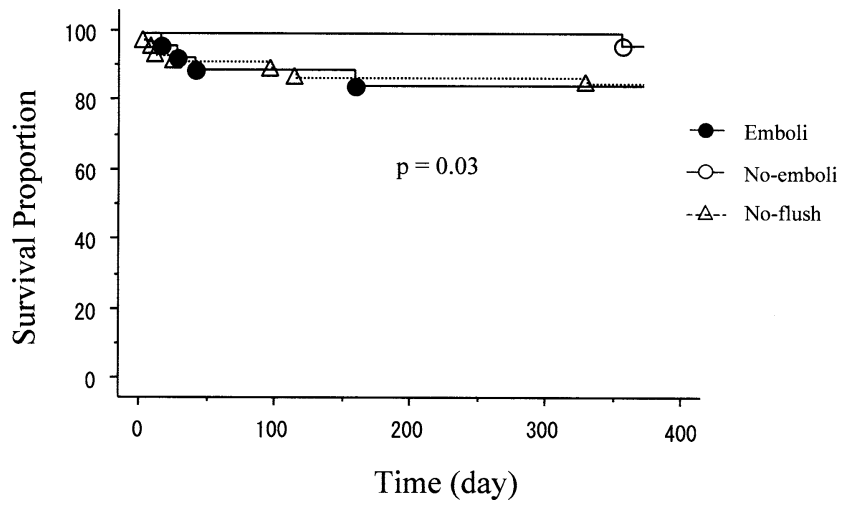

Figure E2. One-year survival. Comparison was made between the emboli and no-emboli groups. 